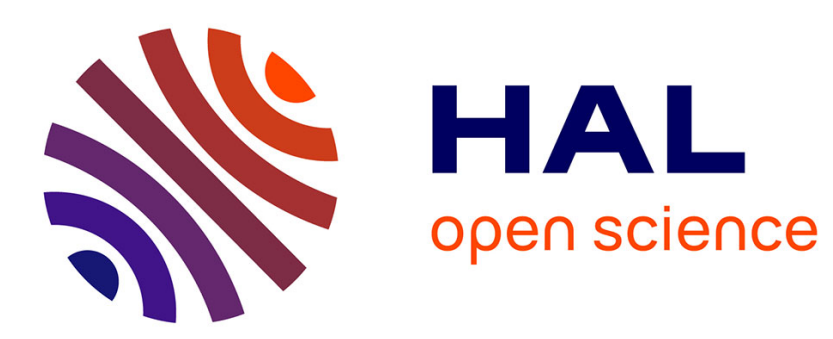

\title{
The kinematics, not the orientation, of an action influences language processing.
}

Sophie-Anne Beauprez, Christel Bidet-Ildei

\section{To cite this version:}

Sophie-Anne Beauprez, Christel Bidet-Ildei. The kinematics, not the orientation, of an action influences language processing.. Journal of Experimental Psychology: Human Perception and Performance, 2018, 44 (11), pp.1712-1726. 10.1037/xhp0000568 . hal-01884047

\section{HAL Id: hal-01884047 \\ https://hal.science/hal-01884047}

Submitted on 7 Jan 2019

HAL is a multi-disciplinary open access archive for the deposit and dissemination of scientific research documents, whether they are published or not. The documents may come from teaching and research institutions in France or abroad, or from public or private research centers.
L'archive ouverte pluridisciplinaire HAL, est destinée au dépôt et à la diffusion de documents scientifiques de niveau recherche, publiés ou non, émanant des établissements d'enseignement et de recherche français ou étrangers, des laboratoires publics ou privés. 
The kinematics, not the orientation, of an action influences language processing Beauprez Sophie-Anne \& Bidet-Ildei Christel

Université de Poitiers; Université François Rabelais de Tours; Centre National de la Recherche Scientifique; Centre de Recherches sur la Cognition et l'Apprentissage (UMR 7295), Poitiers, France

Corresponding Author: Christel Bidet-Ildei (christel.bidet@univ-poitiers.fr) CeRCA/MSHS, Bâtiment A5

5, rue Théodore Lefebvre

TSA 21103

86073 Poitiers cedex 9

Phone: 33 (0)5 49454697

Word count: 8576 
Abstract:

Consistent with the embodied view of cognition, several studies have shown a link between action and the processing of action verbs. However, it is largely unknown how action properties can influence semantic activation during word processing. Based on the observation of point-light displays, the present study addressed this issue. Through three experiments, we assessed whether kinematics and orientation, which are two crucial characteristics of human action, can influence the link between action and language. Participants performed a semantic decision task involving action and nonaction verbs after seeing a point-light display representing either a biological or a nonbiological human movement, the kinematics (Experiments 1 and 2) or orientation (Experiment 3) of which was modified. Experiment 1 showed that important modifications to the kinematics of actions have a direct influence on the link between action and language. Experiment 2 confirmed this effect and showed that the effect is somatotopic because only modifications to the relevant part of actions affected the link between action and language. In contrast, Experiment 3 showed that modifying the orientation did not disturb the influence of an action on language probably due to the use of a mental rotation strategy. Experiment 4 confirmed this view by demonstrating the use of mental rotation when participants have to recognize rotated pointlight actions. These outcomes shed light on how action properties can influence action verbs processing, a crucial step to better understanding the link between action and language.

Keywords: Point-light displays, Biological movement, Kinematics, Orientation, Action verb processing; somatotopy; mental rotation.

Public significant statement: Since the beginning of the 21st century, numerous studies have shown the existence of links between the comprehension of an action verb and the action described in this verb. However, the mechanisms that relies both activities stay in debate. This article is part of this perspective and aims to assess the role of the properties of action (kinematics, orientation) on this link between action and language. 


\section{Introduction}

In the field of embodied cognition (Barsalou, 1999), a growing number of studies suggest that there is crosstalk between language processes and sensorimotor processes. However, despite the large number of studies devoted to this subject, many questions remain unsolved, such as how action is able to influence language processes and, more specifically, what properties of an action are crucial to enable this link between action and language. The aim of this paper is to answer these questions by focusing on two crucial parameters characterizing human movement: kinematics and orientation.

Since the late 1990s, a large number of experiments have clearly demonstrated a link between action and language. At a behavioral level, for example, it was shown that the production of movement was modified when participants simultaneously performed a lexical decision task implying action verbs (Boulenger et al., 2006). It has been suggested that this action-language relationship can be explained by the intervention of the sensorimotor system during language comprehension and production (see Fischer \& Zwaan, 2008; Jirak, Menz, Buccino, Borghi, \& Binkofski, 2010; for reviews). Indeed, brain imaging studies demonstrated the intervention of motor areas during language processing, using functional magnetic resonance imaging (Aziz-Zadeh \& Damasio, 2008; Aziz-Zadeh, Wilson, Rizzolatti, \& Iacoboni, 2006; Hauk, Johnsrude, \& Pulvermüller, 2004), magnetoencephalography (Klepp et al., 2014), electroencephalography (Mollo, Pulvermüller, \& Hauk, 2016), and transcranial magnetic stimulation (Kuipers, van Koningsbruggen, \& Thierry, 2013). In the latter, repetitive stimulation of motor cortices impaired the semantic integration of action verbs (Kuipers et al., 2013). Interestingly, the involvement of the motor system during language processing is somatotopic (Hauk et al., 2004; Tettamanti et al., 2005). For example, Hauk and collaborators (2004) compared the brain activity of participants performing movements or reading action 
verbs related to the hand ("to pick"), feet ("to kick") or face ("to lick"), and brain activity was found to overlap between the passive reading of action verbs related to a specific body part and the actual movement of the body part. Recent behavioral (Bidet-Ildei, Meugnot, Beauprez, Gimenes, \& Toussaint, 2017) and patient studies (Roberts et al., 2017) confirmed this somatotopic effect. Bidet-Ildei and her colleagues (2017) showed that short-term sensorimotor deprivation perturbed specifically the processing of action verbs related to the immobilized limb. In the same way, Roberts et al. (2017) demonstrated that parkinsonian patients with greater upper limb motor impairments responded more slowly to hand verbs than to foot verbs.

Recently, researchers focused on the factors that could influence this action-language relationship. More particularly, they investigated the role of the semantic context provided by language to determine whether the activation induced by language is relevant to and necessary for action language processing or whether it is simply an epiphenomenon. In their study, Zwaan and Taylor (2006) demonstrated that comprehending sentences about manual rotation produced an effect on actual motor responses. This result was interpreted by the authors as evidence of motor resonance involvement. Moreover, they showed that this motor resonance could be modulated by sentence comprehension. That is, manual responses were faster when the rotation performed was in the same direction as the rotation described in the sentence. In another study (Taylor \& Zwaan, 2008), the same authors used adverbs in sentences to shift or maintain participants' attention in relation to the action. When attention was not focused on the action itself, no facilitation effect on compatible motor responses was found. This difference between the two conditions suggests that motor resonance evoked by action word processing is dependent on attentional focus. Similarly, a decrease in the strength of the action-language relationship has also been noted when the semantic context involved focusing attention on the mental state of the agent rather than the action itself (Aravena et al., 2014) or 
when the action was in a negative form instead of a positive form (Aravena et al., 2012). Along the same lines, van Dam, van Dijk Bekkering and Rueschemeyer (2012) investigated the effect of focusing on the visual properties instead of the action properties of an object in an fMRI study. They found that the motor activation related to language was contextdependent. These studies provided evidence that motor structure recruitment relies on specific conditions such as the context of the sentence.

Overall, these studies showed that there are common mechanisms between action and language processing but that the presence of an action word is not in itself sufficient to trigger motor resonance. However, these studies focused on the parameters of the sentence that could modulate the action-language relationship and, until now, no study has considered the role of action properties in this link. Thus, the aim of this paper is to assess this question and, more particularly, to understand whether semantic activation during action word processing can be modified by the properties of an action.

In the literature concerning the effect of action on language processing, there is one important limitation explaining why this question has still not been specifically considered: the majority of studies have focused on action execution. The use of an action execution paradigm hinders the possibility to assess specifically the central processes implied in the action-language relationship because it does not allow differentiation between the influence of central processes and the influence of peripheral processes. However, it is known that peripheral processes can play a role in the action-language relationship. For example, it has been demonstrated that language can produce modification at a peripheral level. In a study by Frak and his colleagues (2010), the participants showed increased grip force when listening to manual action verbs compared with nouns. In the same vein, Aravena and her colleagues (2012) found an enhancement in grip force when their participants listened to affirmative 
sentences containing hand-action verbs, confirming that peripheral processes (such as the execution of a gesture) can be modulated by language processes.

A possible way to avoid the confounding effects of peripheral and central processes is to use action simulation (asking people to imagine themselves or others performing an action) or action observation since, in both cases, no actual movement is required. Several studies have shown that action observation and action simulation, similar to action production, are strongly related to action verb processing. For instance, it has been demonstrated that processing action verbs increases the capacity to recognize point-light actions embedded in a mask composed of moving dots (Bidet-Ildei, Gimenes, Toussaint, Almecija, \& Badets, 2016; Bidet-Ildei, Sparrow, \& Coello, 2011) and, furthermore, increases the capacity to anticipate the end of a perceived action (Springer \& Prinz, 2010). Reciprocally, some studies have shown that action observation can modulate language processing (Beauprez \& Bidet-Ildei, 2017; Liepelt, Dolk, \& Prinz, 2012). For example, Liepelt and his colleagues (2012) showed that participants were faster to produce a word ("close" or "open") when observing beforehand the congruent action (a closed hand or an open hand respectively).

Using action observation presents a second major advantage in that it offers the ability to modify the movement. The perfect example is the technique of point-light displays (Johansson, 1973). In this technique, movements are represented only by points of light located on the main articulations of the body. Despite the poverty of this kind of stimuli, the capacity to detect biological motion in a point-light display is particularly robust. Even when the point-light displays are embedded among many moving dots, people are still able to detect these actions easily (Bertenthal \& Pinto, 1994). By manipulating the dots of the point-light displays, it is possible to modify the movement as one pleases. Several aspects of biological movement have been explored through this methodology. In particular, studies have investigated the consequences of spatial and/or temporal modifications, focusing on the effect 
of such modifications on the perception of biological movement (e.g., Bidet-Ildei, Kitromilides, Orliaguet, Pavlova, \& Gentaz, 2014; Hirai, Senju, Fukushima, \& Hiraki, 2005; Pavlova \& Sokolov, 2000; Sumi, 1984). Globally, these studies demonstrated that perceiving biological motion is dependent on specific spatial and temporal properties. However, these studies focused on how the modification of the biological movement affects the judgment of biological movement, and, thus far, there has been no research considering whether these modifications can also affect higher-order cognitive processing such as language.

Recently, using this point-light display technique, we have shown that perceiving biological motion affects the subsequent processing of action verbs by shortening the time required to perform a semantic decision task implying congruent action verbs (Beauprez \& Bidet-Ildei, 2017). In the present studies, we aim to assess whether this effect would still be present when distortions were introduced into point-light displays, with the goal of understanding how action properties influence subsequent processing of action verbs. We proposed to explore the role of kinematics and orientation which are two important parameters characterizing biological movements (e.g., Pavlova \& Sokolov, 2000; Runeson \& Frykholm, 1981). The main hypothesis behind was that if kinematics or orientation of a movement is crucial to evoke the link between action and language, then modification of the parameter will eliminate or at least disturb the influence of observing this action on language. Therefore, contrary to our previous study (Beauprez \& Bidet-Ildei, 2017), no facilitation effect should be found for congruent action verbs, i.e., the response times for congruent and incongruent verbs should be similar. By contrast, if the parameter does not play a role in the action-language relationship, then its modification will have no effect, and the results of watching the modified movement should be similar results to the results of observing a biological motion. In this case, a facilitation effect on the time to process congruent action 
verbs should still be found, i.e., the response times for congruent verbs should be shorter than those for incongruent verbs.

\section{Experiment 1: role of action kinematics in the processing of action verbs}

A great number of studies have shown that kinematics is a very important parameter of human movements (see Blake \& Shiffrar, 2007 for a review). Through kinematics, humans are able to recognize and distinguish many action characteristics. For example, through kinematics alone, people are able to determine whether an action is directed toward the left or the right side (Davila, Schouten, \& Verfaillie, 2014). They can also detect characteristics such as the sex (Kozlowski \& Cutting, 1977), identity (Beardsworth \& Buckner, 1981; Cutting \& Kozlowski, 1977; Loula, Prasad, Harber, \& Shiffrar, 2005), emotional state (Atkinson, Dittrich, Gemmell, \& Young, 2004; Chouchourelou, Matsuka, Harber, \& Shiffrar, 2006; Clarke, Bradshaw, Field, Hampson, \& Rose, 2005)and intention (Orliaguet et al., 1997; Martel, Bidet-Ildei, \& Coello, 2011) of the actor and even determine some characteristics of the objects with which the actor interacts, such as their weight (Runeson \& Frykholm, 1981). Moreover, modifying the kinematics of motion disturbs the recognition of a movement (Bidet-Ildei, Méary, \& Orliaguet, 2006; Pavlova, Krägeloh-Mann, Birbaumer, \& Sokolov, 2002) and underlies some visual illusions (Viviani \& Stucchi, 1989). Kinematics modifications also have a direct consequence for the capacity to anticipate action (Martel, Bidet-Ildei, \& Coello, 2011; Pozzo, Papaxanthis, Petit, Schweighofer, \& Stucchi, 2006) and can modify the activation of the motor system during action observation (Bouquet, Gaurier, Shipley, Toussaint, \& Blandin, 2007). Moreover, this sensitivity to kinematics appears early in development. Indeed, 5-month-old children discriminate between a point-light walker 
whose local rigidity is maintained from one whose local rigidity is modified (Bertenthal, Proffitt, \& Kramer, 1987), and even from the age of three days, neonates can distinguish biological motions from motions with non-biological kinematics (Méary, Kitromilides, Mazens, Graff, \& Gentaz, 2007).

Considering these results, it appears that kinematics is a very important characteristic for action observation. Recently, it has been shown that kinematics is also able to affect higher-order cognitive processing such as abstract conceptual thought (Badets, Bidet-Ildei, \& Pesenti, 2015). In the aforementioned study, Badets and his colleagues have shown that pointing movements with biological kinematics induced space-number bias (Dehaene, 1992) in a random-number generation task whereas this effect disappeared when pointing movements had non-biological kinematics.

Thus, the present experiment examined whether kinematics, a crucial characteristic of human action, can affect the link between action and language. Participants performed an action verb processing task after the presentation of point-light sequences representing human movements with biological or non-biological kinematics. We hypothesized that nonbiological kinematics should disrupt the usual link relating action observation and action verbs processing. Specifically, point-light actions with non-biological kinematics should be ineffective, or at least less effective, than actions with biological kinetics, at improving subsequent reaction times to judge congruent action verbs

\section{Method}

\subsection{Participants}

The a priori calculation of our sample size was made with G*Power software (Faul, Erdfelder, Lang, \& Buchner, 2007). The calculation was based on a repeated-measures ANOVA design from the results obtained in a previous work (Beauprez \& Bidet-Ildei, 2017, 
Cohen's $d=1.12$, correlations between repeated measures $=0.5$ ). Statistical significance was set at $p<.05$ and the power at 0.90 . The results indicated that 10 participants would be sufficient to provide an estimated power of 0.90 . However, our previous work included only one comparison, whereas three comparisons were included in the present study. Therefore, we decided to include 30 participants in this first study. Thus, 30 French-speaking university students (16 male, 27 right-handed) aged 18 to 28 years $(M=19, S D=2.44$ years) participated in this experiment. They were recruited in exchange for course credit. All participants had normal or corrected-to-normal vision and had no history of motor, perceptual or neurological disorders. Moreover, all participants provided written informed consent prior to their inclusion in the experiment. They were also naïve to the purpose of the study.

\subsection{Apparatus}

The participants sat on a chair in front of a table in a dimly lit room. A computer screen (spatial resolution of 1280 pixels $* 800$ pixels and temporal resolution of $60 \mathrm{~Hz}$ ) was on the table. A response box was placed on the table between the participants and the computer screen so that the participants could easily provide their responses by pressing the button associated with the answer "yes" or "no".

\subsection{Primes and Stimuli}

\subsubsection{Primes: Point-light displays (PLD)}

The videos used as primes were point-light displays (Johansson, 1973). These videos were recorded using a motion capture system consisting of multiple optoelectronic cameras (Vicon motion system; see http://www.vicon.com for further information). We recorded and used 15 different human actions: applauding, climbing (a ladder), falling, getting off (a ladder), jumping, moving back, pedaling, picking up, pushing, putting down, running, scratching, 
sweeping, turning, and walking. The video included 13 points of light located on the main body joints (shoulders, elbows, wrists, hips, knees and ankles) and the head. From these 15 human actions, we created 3 types of PLD:

- Biological movement corresponds to a recorded PLD with no modification, i.e., a natural movement of a human being.

- Constant kinematics corresponds to a modification of the biological PLD so that the velocity of the different points maintains a constant norm throughout the movement.

- Inverted kinematics corresponds to a modification of the biological PLD so that the norm velocity of the different points is inverted with respect to the mean norm original velocity (see figure 1 for an illustration of these modifications).

These modifications affect the velocity of the different dots while maintaining the original path of the dots and the original movement duration. They were made using PLAViMoP, a new software program allowing the visualization and modification of PLDs (PLAViMoP is under development and will be available online in the near future; see http://cerca.labo.univpoitiers.fr/projets-realises-au-cerca-fr/plavimop-point-light-action-visualization-and-

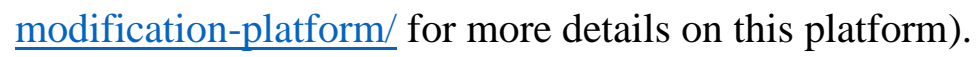

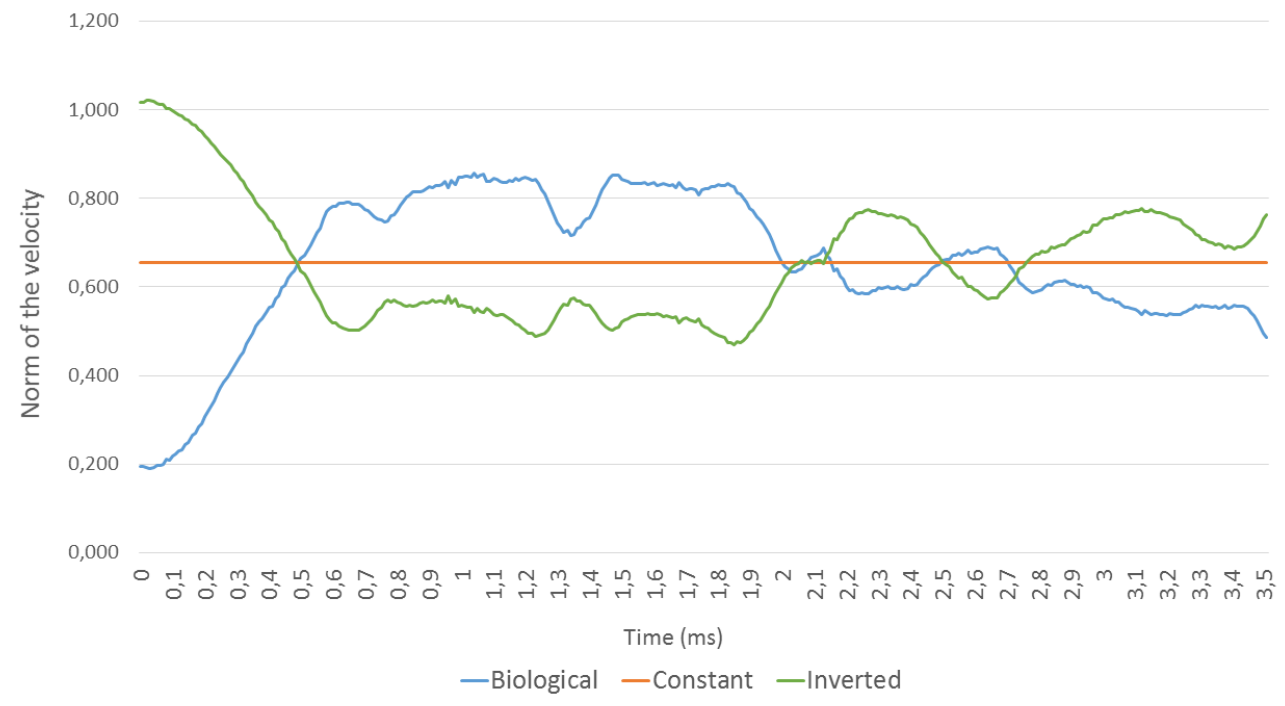

Fig. 1: Mean norm of the velocity for the biological, constant and inverted "Move back" 
PLDs from the beginning $(0 \mathrm{~ms})$ to the end of the movement $(3.5 \mathrm{~ms})$.

\subsubsection{Stimuli: verbs}

Thirty verbs were used. Half of them were "action verbs", i.e., verbs describing a movement of the body (e.g., to jump, to walk), while the other half were "non-action verbs" (e.g., to think, to want). These non-action verbs were not analyzed. They were included only to develop a task for participants (see appendix 1 for the entire list of verbs). All verbs were presented in French in the infinitive form.

The action verbs could be congruent or incongruent with the PLD presented as a prime. An example of a congruent trial is seeing the PLD of the walking movement before reading the word "to walk", whereas an example of incongruent trial is seeing the PLD of the walking movement before reading the word "to jump".

\subsection{Procedure}

Each trial used the following procedure: first, a fixation cross appeared (500 ms); then, the prime PLD (2500 ms on average) appeared; and finally, following another fixation cross (500 $\mathrm{ms}$ ), the stimulus (a verb) appeared. The stimulus stayed on the screen until the participant entered a response. The task of the participant was to judge, as quickly and accurately as possible, whether the word that was presented was an action verb. The "yes" answer was consistently entered by the participant's dominant hand, whereas the "no" answer was entered by the other hand.

The experimental session was divided into three blocks, and breaks were permitted between blocks. The first block was either the constant or the inverted condition (half of the participants began with the constant condition, and the other half with the inverted condition), and then the second block was the other modified condition. The biological condition was 
always presented at the end so that seeing the biological movement would not influence recognition of the modified PLDs. In all blocks, the presentation order of the trials was randomized across participants.

After the participants completed the experimental task, a short questionnaire was administered to them. The aim of this questionnaire was to verify whether each action in the primes had been recognized by the participants. Each PLD was presented to the participants, who were asked to provide one (or several) verb corresponding, according to them, to the action of the prime. The PLD obtained a score of 1 when the answer provided by the participant corresponded to the action, namely, when the participants gave the exact verb of the action or a semantically close verb (e.g., "to hop" instead of "to jump"). The PLD obtained a score of 0 when the answer provided by the participant differed semantically from the one expected (e.g., "to dance" instead of "to scratch"). Then, a percentage of the recognition rate was calculated.

\subsection{Data Analysis}

Response time and accuracy were recorded. Significant differences were examined by ANOVA, with congruency (congruent action $\mathrm{x}$ incongruent action) and condition (biological $\mathrm{x}$ constant $\mathrm{x}$ inverted) as within-subject factors, and partial eta-squared values were used to report effect sizes. Paired comparisons were performed using the Newman-Keuls procedure. The significance level was fixed at $p<.05$.

\section{Results}

\subsection{Experimental task}

Given the high accuracy rate for each type of stimulus and prime (>98\%), we analyzed only the response times. The analyses showed that response times (see figure 2) varied 
according to condition $\left(\mathrm{F}(2,58)=7.88 ; p<.001 ; \eta_{\mathrm{p}}{ }^{2}=0.21\right)$ and congruency $(\mathrm{F}(1,29)=31.83$; $\left.p<.001 ; \eta_{\mathrm{p}}^{2}=0.52\right)$. Moreover, there was a significant interaction between condition and congruency $\left(\mathrm{F}(2,58)=4.23 ; p=.02 ; \eta_{\mathrm{p}}^{2}=0.13\right)$. The Newman-Keuls post-hoc analyses revealed that in the biological condition, response times for congruent action verbs were shorter than those for incongruent action verbs (569 ms vs $618 \mathrm{~ms}, p<.001)$. Similarly, in the constant condition, response times for congruent action verbs were shorter than those for incongruent action verbs (617 ms vs $655 \mathrm{~ms}, p<.001)$; however, in the inverted condition, response times for congruent and incongruent action verbs were not significantly different (626 ms vs $647 \mathrm{~ms}, p=.11)$.

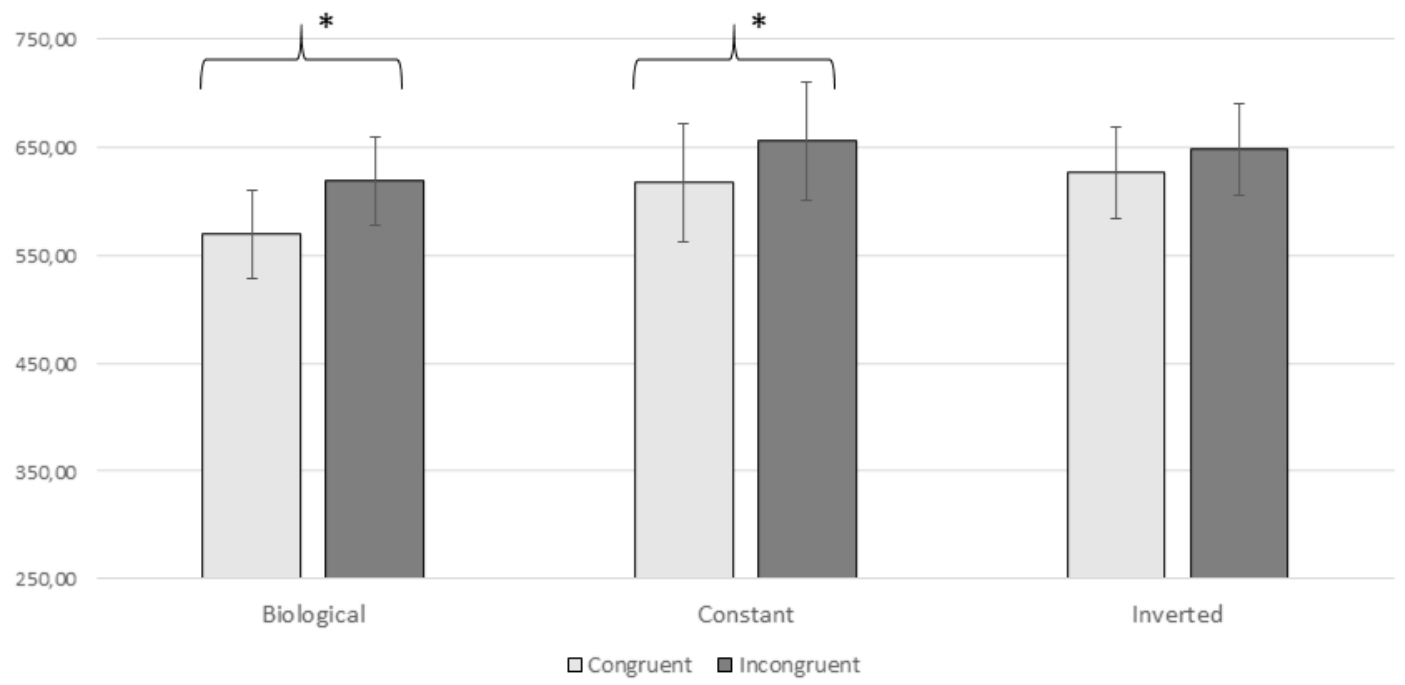

Fig. 2: Mean response times (ms) according to condition (biological, constant, inverted) and congruency (congruent and incongruent). The error bars indicate the $95 \%$ confidence interval. An asterisk indicates a significant difference $(p<.05)$.

In summary, there was a facilitation effect (shorter response times for congruent action verbs than for incongruent action verbs) in the biological and constant conditions but not in the inverted condition. Student's t-test revealed that this facilitation effect was significantly stronger $(p=.03)$ in the biological condition $(56 \mathrm{~ms})$ than in the inverted condition $(16 \mathrm{~ms})$. 
The effect in the constant condition was intermediate $(42 \mathrm{~ms})$, since it was not different from either the biological condition $(p=.19)$ or the inverted condition $(p=.07)$.

\subsection{Recognition questionnaire}

The results of the questionnaire on recognition of the PLDs showed a difference according to the type of PLD $(\mathrm{F}(2,58)=0.05 ; p<.001)$. Biological PLDs were better recognized $(96 \%)$ than the constant or inverted PLDs ( $p<.001$ each). However, the recognition rates were similar between the constant (90\%) and inverted (88\%) PLDs $(p=.07)$.

\section{Discussion}

The aims of this first study were as follows: (1) to replicate our previous results, i.e., demonstrate a facilitation effect for action verb processing following the visual presentation of a congruent action (Beauprez \& Bidet-Ildei, 2017), and (2) to assess whether the kinematics of actions can modulate this effect and therefore the action-language relationship. To do so, we compared priming effects obtained in action verb processing when the action presented as a prime had biological, constant or inverted kinematics. Our results confirmed that perceiving a biological action facilitates the subsequent processing of a congruent action verb (Beauprez \& Bidet-Ildei, 2017). Participants were indeed faster to answer when the action of the prime and the action of the verbs were congruent. Moreover, we demonstrated that this facilitation was dependent on the kinematics of the action presented as prime. Indeed, this facilitation effect was found in the constant condition, but disappeared in the inverted condition.

Thus, it appears that the influence of action observation on language processing is dependent on the kinematics of the action observed. Biological kinematics optimizes the link between action observation and action verb processing and this link decreases or disappears 
when the perceived kinematics modify the biological characteristics of the movement. This finding confirms the role of biological kinematics in higher-order cognitive processing (Badets et al., 2015) and demonstrates for the first time its implication in the link between action observation and language. Interestingly, it seems that the degree of change in kinematics can influence the action-language relationship. Indeed, whereas the facilitation effect disappeared in the inverted condition (large change in velocity), it merely decreased in the constant condition (small change in velocity).

One explanation could be that the difference between the constant and inverted conditions is related to explicit recognition of the action. However, the analysis of the questionnaire did not confirm this view because there was no difference between the recognition rates of constant and inverted point-light actions. Moreover, through discussion with the participants after the experiment, we realized that most of them did not even notice that there were differences between the point-light displays presented in the biological, constant and inverted conditions, suggesting that the effect obtained relies on implicit mechanisms. One alternative possibility could be that human action perception is robust against small changes in kinematics. Previous results have supported this possibility by showing that visual preference judgment of handwriting movements was affected only when the kinematics of handwriting had undergone major modification (Bidet-Ildei, KitromilidesSalerio, Orliaguet \& Badets, 2011).

\section{Experiment 2: action kinematics and somatotopy}

The results of Experiment 1 demonstrated for the first time that the kinematics of an action is a property of the action that influences the effects of embodied language processing. Following this, one can ask whether the disappearance of the facilitation effect could be 
related to somatotopy. Indeed, we know that the involvement of the motor system during language processing is somatotopic (Hauk et al., 2004; Tettamanti et al., 2005). Therefore, we could expect that it is specifically the modification of the kinematics of the effector involved in the verb that causes the results obtained in Experiment 1. However, the PLD we used does not allow us to answer this question since the kinematics of all points of the PLD were modified. Thus, we designed a second experiment with a similar procedure. The first aim of this study was to replicate the results of Experiment 1 by showing that modifying the kinematics of an action leads to the disappearance of the facilitation effect. The second aim of this study was to determinate whether the absence of an action-language relationship is specifically linked to the modification of the specific effector involved in the verb. If the effect of the kinematics obtained in Experiment 1 is somatotopic, we hypothesized that modification of the kinematics of all articulations (inverted PLD) or modification of the kinematics of articulations related to a relevant part of an action (PLD with inverted kinematics of only the main effector) should not improve subsequent reaction times in judging congruent action verbs. In contrast, if the kinematics of the main effector are conserved (biological PLD or PLD with a modification of another effector), then the action-language relationship should be observed.

\section{Method}

\subsection{Participants}

The same number of participants was recruited as that in Experiment 1; thus, 30 new French-speaking university students (19 male, 25 right-handed) aged 18 to 28 years $(M=20$, $\mathrm{SD}=2.73$ years) participated in this second experiment. The participants were recruited in exchange for course credit. All participants had normal or corrected-to-normal vision and had no history of motor, perceptual or neurological disorders. Moreover, all participants provided 
written informed consent prior to their inclusion in the experiment. The participants were also naïve to the purpose of the study.

\subsection{Primes, Stimuli and Procedure}

\subsubsection{Primes: Point-light displays (PLDs)}

We used 10 different human actions from Experiment 1. Five were actions involving the upper part of the body (applauding, picking up an object, pushing, putting down an object and scratching), and the other five were actions involving the lower part of the body (jumping, moving back, pedaling, running and walking). From these 10 actions, we created 4 types of PLD by using PLAViMoP:

- Biological movement, as in Experiment 1, corresponded to recorded PLD with no modifications, i.e., a natural movement of a human being.

- Total inverted kinematics, as in Experiment 1, corresponded to a modification of the biological PLD so that the normal velocity of all points was inverted with respect to the mean normal original velocity.

- Inverted kinematics on the relevant effector: inverted kinematics modifications were applied but only on the 6 points of light representing the articulations mainly involved in the action. For the upper limb actions, the inverted modification was applied to the wrists, elbows and shoulders. For the lower limb actions, the inverted modification was applied to the ankles, knees and hips.

- Inverted kinematics on an irrelevant effector: inverted kinematics modifications were applied but only on the 6 points of light of the articulations that were not mainly involved in the action. For the upper limb actions, the inverted modification was applied to the ankles, knees and hips. For the lower limb actions, the inverted 
modification was applied to the wrists, elbows and shoulders (see Table 1 for a summary of the modifications).

Table 1: modifications applied to the different points of the PLD according to the 4 types of PLD used in Experiment 2.

\begin{tabular}{|c|c|c|c|c|}
\hline & $\begin{array}{c}\text { Biological } \\
\text { PLD }\end{array}$ & Inverted PLD & $\begin{array}{c}\text { Relevant effector } \\
\text { inverted PLD }\end{array}$ & $\begin{array}{c}\text { Irrelevant effector } \\
\text { inverted PLD }\end{array}$ \\
\hline Upper limb actions & $\begin{array}{c}\text { No } \\
\text { modification }\end{array}$ & $\begin{array}{c}\text { All points of } \\
\text { light }\end{array}$ & $\begin{array}{c}\text { Wrists, elbows, } \\
\text { shoulders }\end{array}$ & $\begin{array}{c}\text { Ankles, knees, } \\
\text { hips }\end{array}$ \\
\hline Lower limb actions & $\begin{array}{c}\text { No } \\
\text { modification }\end{array}$ & $\begin{array}{c}\text { All points of } \\
\text { light }\end{array}$ & $\begin{array}{c}\text { Ankles, knees, } \\
\text { hips }\end{array}$ & $\begin{array}{c}\text { Wrists, elbows, } \\
\text { shoulders }\end{array}$ \\
\hline
\end{tabular}

\subsubsection{Stimuli and procedure}

As in Experiment 1, the stimuli were action verbs or nonaction verbs. There were 10 action verbs corresponding to the action of the prime (e.g., to jump, to put down an object) and 10 non-action verbs (e.g., to need, to have) that were only included to develop a task for the participants. All verbs were presented in French in the infinitive form.

The procedure was exactly the same as that of Experiment 1, except that it contained 4 blocks instead of 3 . The first three blocks were the total inverted condition, the relevant effector inverted condition and the irrelevant effector inverted condition. The order of the blocks was randomized across participants. However, the biological condition was always presented at the end so that seeing the biological movement would not influence the recognition of the modified PLD.

After the participants completed the experimental task, the same short recognition questionnaire used in Experiment 1 was administered to the participants. 


\subsection{Data Analysis}

Response time and accuracy were recorded. Significant differences were examined by ANOVA, with congruency (congruent action $\mathrm{x}$ incongruent action) and condition (biological $\mathrm{x}$ inverted $\mathrm{x}$ relevant effector $\mathrm{x}$ irrelevant effector) as within-subject factors, and the partial eta-squared values were used to report effect sizes. Paired comparisons were performed using the Newman-Keuls procedure. The significance level was fixed at $p<.05$.

\section{Results}

\subsection{Experimental task}

The analyses showed that the response times (see figure 3) varied according to condition $\left(\mathrm{F}(3,87)=4.16 ; p=.008 ; \eta_{\mathrm{p}}^{2}=0.13\right)$ and congruency $\left(\mathrm{F}(1,29)=14.87 ; p<.001 ; \eta_{\mathrm{p}}{ }^{2}=0.34\right)$. Moreover, there was a significant interaction between condition and congruency $(\mathrm{F}(3,87)=$ 3.72; $p=.01 ; \eta_{\mathrm{p}}^{2}=0.11$. The Newman-Keuls post hoc analyses revealed that in the biological condition, the response times for congruent action verbs were shorter than those for incongruent action verbs (605 ms vs $657 \mathrm{~ms}$, respectively, $p=.003$ ). Similarly, in the irrelevant effector condition, the response times for congruent action verbs were shorter than those for incongruent action verbs $(630 \mathrm{~ms}$ vs $695 \mathrm{~ms}$, respectively, $p<.001)$. However, the response times for congruent and those for incongruent action verbs were not significantly different in the inverted condition (671 ms vs $684 \mathrm{~ms}$, respectively, $p=.38$ ) or in the relevant effector condition (693 ms vs $697 \mathrm{~ms}, p=.96$ ). 


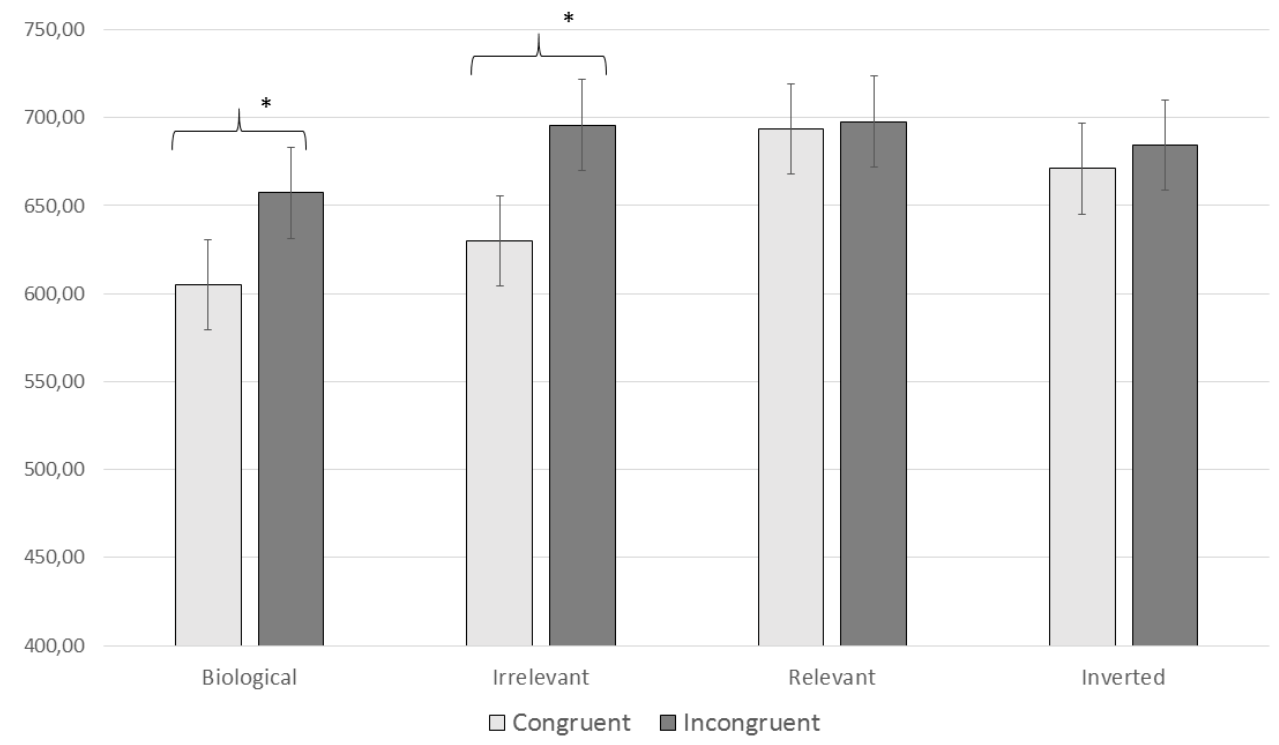

Fig. 3: Mean response times (ms) according to condition (biological, inverted, relevant effector, and irrelevant effector) and congruency (congruent and incongruent). The error bars indicate the $95 \%$ confidence interval. An asterisk indicates a significant difference $(p<.05)$.

\subsection{Recognition questionnaire}

The results of the recognition questionnaire regarding the PLDs showed a difference according to the types of PLD $(\mathrm{F}(3,87)=5.13 ; p=.003)$. The biological PLDs were better recognized $(96 \%)$ than the modified PLD $(p<.05$ each). However, the recognition rates were similar when modifications were made to all points (90\%), when the modification was to the main effector only $(91 \%)$ and when the modification was to another effector $(93 \%, p>.07$ each).

\section{Discussion}

The first aim of this second study was to replicate the results of Experiment 1, i.e., to demonstrate that the facilitation effect observed for congruent action verbs when they appeared after point-light biological movement disappears when the perceived movement 
presents important kinematic modifications. The results obtained in Experiment 2 confirmed this finding by demonstrating once more that the participants were quicker to respond to congruent action verbs in the biological condition, but this facilitation effect was not found when observing PLD with inverted kinematics. The second aim of Experiment 2 was to identify whether this effect is somatotopic. Namely, we wanted to determine whether it is specifically the main effector involved in the action that determines the influence of action observation on action verb processing. Interestingly, the results of Experiment 2 showed that there was no influence of action perception on language processing when the modification to the kinematics was applied to the main effector, whereas the facilitation effect was still observed when the kinematic modification was applied to a part of the body that was not relevant for the action. These results were in agreement with the idea that the involvement of sensorimotor processing during language processing is somatotopic (Hauk et al., 2004; Tettamanti et al., 2005) and showed for the first time that the link between action and language is specifically based on kinematic information mainly related to the body part involved in an action.

\section{Experiment 3: role of action orientation in the processing of action verbs}

The second parameter we chose to study was orientation. Previous works have shown that the visual perception of human movement is vulnerable to orientation modifications. Shiffrar and his colleagues (1997) showed that only point-light walkers with a canonical orientation (normal orientation) were recognized, while walkers with a $90^{\circ}$ or $180^{\circ}$ rotation were not. In the same vein, Pavlova and Sokolov (2000) used rotation of point-light displays to study how orientation affects the perception of biological motion, and they showed that the recognition of these displays was impeded by non-canonical orientation, even when 
participants had been previously familiarized with the rotated point-light displays (recognition rate below $40 \%$ for the $90^{\circ}$ walker and close to $0 \%$ for the $180^{\circ}$ walker).

Interestingly, this orientation sensibility is also found in children. From birth, newborn babies show a preference for point-light displays presented in a canonical orientation (Bardi, Regolin, \& Simion, 2014; Simion, Regolin, \& Bulf, 2008). Moreover, several animal species also show a preference for biological orientation (Blake, 1993; Oram \& Perrett, 1994; Parron, Deruelle, \& Fagot, 2007; Schluessel, Kortekamp, Cortes, Klein, \& Bleckmann, 2015) suggesting that this sensitivity is genetically programmed.

Together, these studies seem to indicate that orientation is a determinant of biological motion perception. Thus, it might be hypothesized that modifying of the orientation of a point-light display would disrupt the action-language relationship.

\section{Method}

\subsection{Participants}

Thirty French-speaking university students (21 male, 25 right-handed) aged 18 to 24 years $(M=20, S D=1.76$ years $)$ participated in this experiment. They were recruited in exchange for course credit. All participants had normal or corrected-to-normal vision and had no history of motor, perceptual or neurological disorders. Moreover, all participants provided written informed consent prior to their inclusion in the experiment. They were also naïve to the purpose of the study.

\subsection{Apparatus, Stimuli, Primes and Procedure}

The experiment had exactly the same design as experiment 1 and 2 (the kinematics studies). The only difference concerned the PLDs used as primes. We used the same actions, 
but the modification applied to these PLDs concerned orientation instead of kinematics. Thus, there were 3 types of PLD (see figure 4 for an illustration of the modification):

- Biological movement corresponds to a recorded PLD with no modification, i.e., a natural movement of a human being in a canonical orientation.

- Minor rotation $\left(90^{\circ}\right)$ corresponds to a $90^{\circ}$ clockwise rotation biological PLD.

- Major rotation $\left(180^{\circ}\right)$ corresponds to a $180^{\circ}$ clockwise rotation of the biological PLD.

These modifications were also made using PLAViMoP. They affected only the orientation and did not modify the kinematic parameters.

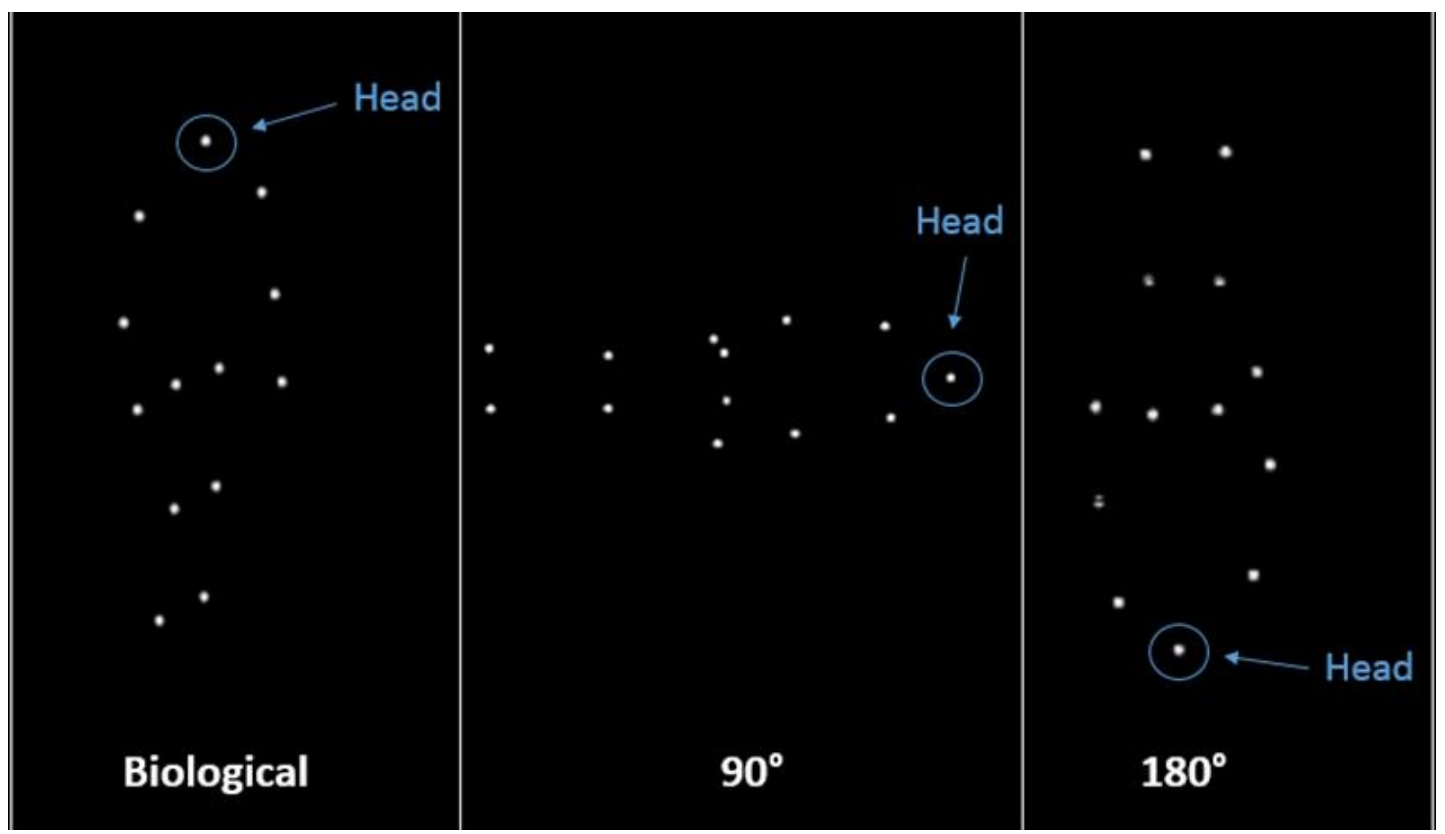

Fig. 4: Example of PLD with a biological orientation or a modified orientation $\left(90^{\circ}\right.$ and $180^{\circ}$ clockwise rotation).

\subsection{Data Analysis}

Response times and accuracy were recorded. Significant differences were examined by ANOVA, with congruency (congruent action $\mathrm{x}$ incongruent action) and condition (biological x $90^{\circ} \times 180^{\circ}$ ) as within-subject factors, and partial eta-squared values were used to report 
effect sizes. Paired comparisons were performed using the Newman-Keuls procedure. The significance level was fixed at $p<.05$.

\section{Results}

\subsection{Experimental task}

Again, a high accuracy rate was obtained for each type of stimulus and prime (>97\%); thus only the response times were analyzed. The analyses showed that response times (see figure 5) varied according to condition $\left(\mathrm{F}(2,58)=4.24 ; p=.02 ; \eta_{\mathrm{p}}{ }^{2}=0.13\right)$ and to congruency $\left(\mathrm{F}(1,29)=41.99 ; p<.001 ; \eta_{\mathrm{p}}^{2}=0.59\right)$. However, no significant interaction was obtained between condition and congruency $\left(\mathrm{F}(2,58)=0.23 ; p=.79 ; \eta_{\mathrm{p}}{ }^{2}=0.01\right)$.

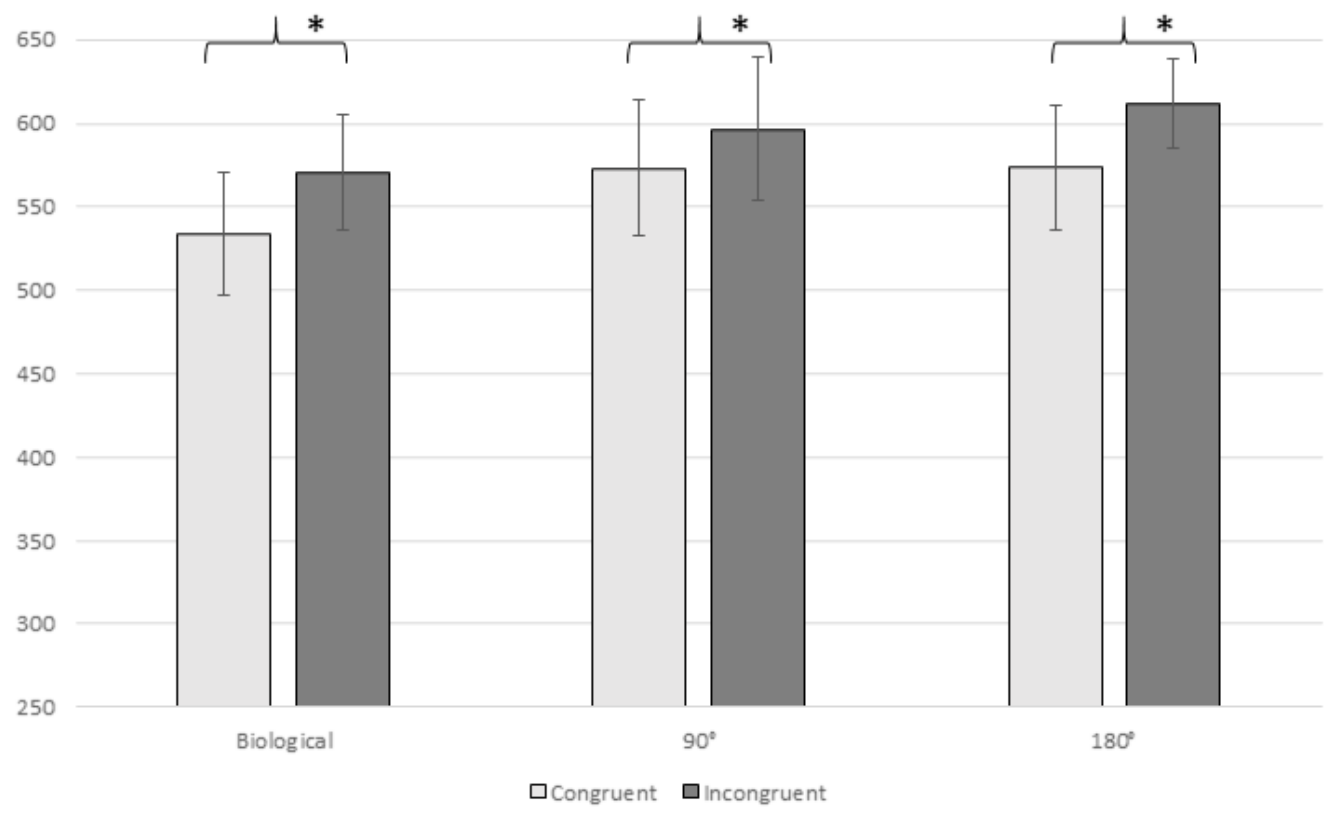

Fig. 5: Mean response times (ms) according to condition (biological, 90, $180^{\circ}$ ) and congruency (congruent and incongruent). The error bars indicate the 95\% confidence interval. An asterisk indicates a significant difference $(p<.05)$.

In summary, the facilitation effect was the same in the biological, $90^{\circ}$ rotation and $180^{\circ}$ rotation conditions ( $36 \mathrm{~ms}, 23 \mathrm{~ms}$ and $38 \mathrm{~ms}$ respectively). 


\subsection{Recognition questionnaire}

The results of the recognition questionnaire about the PLD showed a difference according to the type of $\operatorname{PLD}\left(\mathrm{F}(2,58)=7.08 ; p=.002 ; \eta_{\mathrm{p}}{ }^{2}=0.20\right)$. Biological PLDs were better recognize $(97 \%)$ than the rotated ones $(p<.05$ each). However, the recognition rates were similar for $90^{\circ}(91 \%)$ and $180^{\circ}(88 \%)$ rotated PLDs $(p=.15)$.

\section{Discussion}

The aim of this second study was to assess whether modifying action orientation can affect the action-language relationship. Two different modifications were applied to the orientation of point-light displays: a $90^{\circ}$ rotation and a $180^{\circ}$ rotation. The results confirmed once again that visual presentation of point-light actions facilitates the processing of congruent action verbs (Beauprez \& Bidet-Ildei, 2017) and showed that this effect is robust against changes in orientation. Indeed, the facilitation effect was obtained in every condition.

Thus, contrary to what was expected, it seems that the orientation is not a crucial parameter for the influence of action observation on action verb processing. Indeed, modification of this parameter did not lead to the disappearance of the facilitation effect. One possible explanation could be that participants used systematic mental rotation in our study. In accordance with this interpretation, a recent study showed that observing inverted biological motion activates the left fusiform gyrus (Pavlova et al., 2017), a region known to be engaged in mental rotation (Tomasino \& Gremese, 2015). Moreover, in our study, this strategy was facilitated because the different conditions were presented in different blocks, i.e., all stimuli were presented in the same rotation several times which could have facilitated the recognition of action by participants. The PLDs were also followed by action verbs and participants may have guessed that those actions were depicted in the PLDs. Thus, as a strategy, participants 
could have mentally rotated the point-light displays to recognize the actions. This is in accordance with the level of explicit recognition obtained in our study. In fact, although we found a significant difference between the recognition of biological point-light displays and the recognition of point-light displays with modified orientation, these last stimuli were recognized in more than $92 \%$ of trials, which is a very high level of recognition in comparison with the level of recognition obtained in a previous experiment (Pavlova \& Sokolov, 2000). To more specifically assess whether rotated point-light displays implicitly cause mental rotation, we carried out the last experiment (Experiment 4).

\section{Experiment 4: mental rotation and rotated PLD}

In Experiment 3, we suggested that our participants could have spontaneously used mental rotation to recognize the actions pictured in the PLD. Typically, in the literature, mental rotation has been demonstrated when participants' response times increase with increasing rotational angles between two depictions. When mental rotation is used, a linear relation between the response time and the rotation angle is observed, i.e., the more the participant has to rotate, the longer the mental rotation takes (Corballis \& McMaster, 1996; Kosslyn, DiGirolamo, Thompson, \& Alpert, 1998). Thus, we hypothesized that if mental rotation is spontaneously used when people have to recognize rotated PLD, then we should observe different response times according to the rotations of PLD. The more complex the rotation is, the longer the response time should be. In contrast, if mental rotation is not used in the recognition of rotated PLD, then no differences should be observed regardless of the orientations of the PLD.

\section{Method}




\subsection{Participants}

Thirty French-speaking university students (19 male, 25 right-handed) aged 18 to 26 years $(\mathrm{M}=20, \mathrm{SD}=2.71$ years $)$ participated in this experiment. They were recruited in exchange for course credit. All participants had normal or corrected-to-normal vision and had no history of motor, perceptual or neurological disorders. Moreover, all participants provided written informed consent prior to their inclusion in the experiment. The participants were also naïve to the purpose of the study.

\subsection{Primes and stimuli}

\subsubsection{Primes: $P L D$}

The 15 actions used in experiment 3 (orientation modifications) were used. Five different orientations were used: $0^{\circ}$ (canonical orientation), $45^{\circ}$ rotation, $90^{\circ}$ rotation, $135^{\circ}$ rotation and $180^{\circ}$ rotation. Once again, these modifications were made using PLAViMoP.

\subsubsection{Stimuli: verbs}

Fifteen verbs were used. There were all "action verbs" corresponding to the action of the PLD (to applaud, to climb, to fall, to get off, to jump, to move back, to pedal, to pick up an object, to push, to put down an object, to run, to scratch, to sweep, to turn, and to walk).

\subsection{Procedure}

Each trial used the following procedure: first, a fixation cross appeared (500 ms); then, a verb appeared $(1500 \mathrm{~ms})$; and finally, after another fixation cross $(500 \mathrm{~ms})$, the PLD appeared. The PLD could correspond or not to the verbs. The task of the participants was to recognize the PLD, namely, they had to judge, as quickly and accurately as possible, whether the PLD corresponded to the verb previously seen. All types of the PLD $\left(0^{\circ}, 45^{\circ}, 90^{\circ}, 135^{\circ}\right.$ 
and $180^{\circ}$ rotations) were presented in the same block. The presentation order of the trials was randomized across participants. Breaks were permitted every 50 trials.

\subsection{Data analysis}

Response time and accuracy were recorded. Significant differences were examined by ANOVA, with the degree of rotation $\left(0^{\circ} \times 45^{\circ} \times 90^{\circ} \times 135^{\circ} \times 180^{\circ}\right)$ as the within-subject factor, and partial eta-squared values were used to report effect sizes. Paired comparisons were performed using the Newman-Keuls procedure. The significance level was fixed at $p<$ .05 .

\section{Results}

The analyses showed that the response times (see figure 6) varied according to the degree of rotation $\left(\mathrm{F}(4,116)=47.08 ; p<.001 ; \eta_{\mathrm{p}}^{2}=0.62\right)$. The Newman-Keuls post hoc analyses revealed that the times to respond to the $0^{\circ}$ PLD $(1160 \mathrm{~ms})$ were significantly shorter than those to the $45^{\circ}$ PLD $(1242 \mathrm{~ms}, p<.001)$, which were shorter than those to the $90^{\circ}$ PLD (1313 ms, $p=.002)$, which were shorter than those to the $135^{\circ}$ PLD (1367 ms, $\left.p=.02\right)$, which were shorter than those to the $180^{\circ}$ PLD $(1440 \mathrm{~ms}, p=.001)$. Subsequent polynomial analyses revealed a significant linear increase in the response times with increased rotation angles $(\mathrm{F}(1,29)=153.64 ; p<.001)$. 


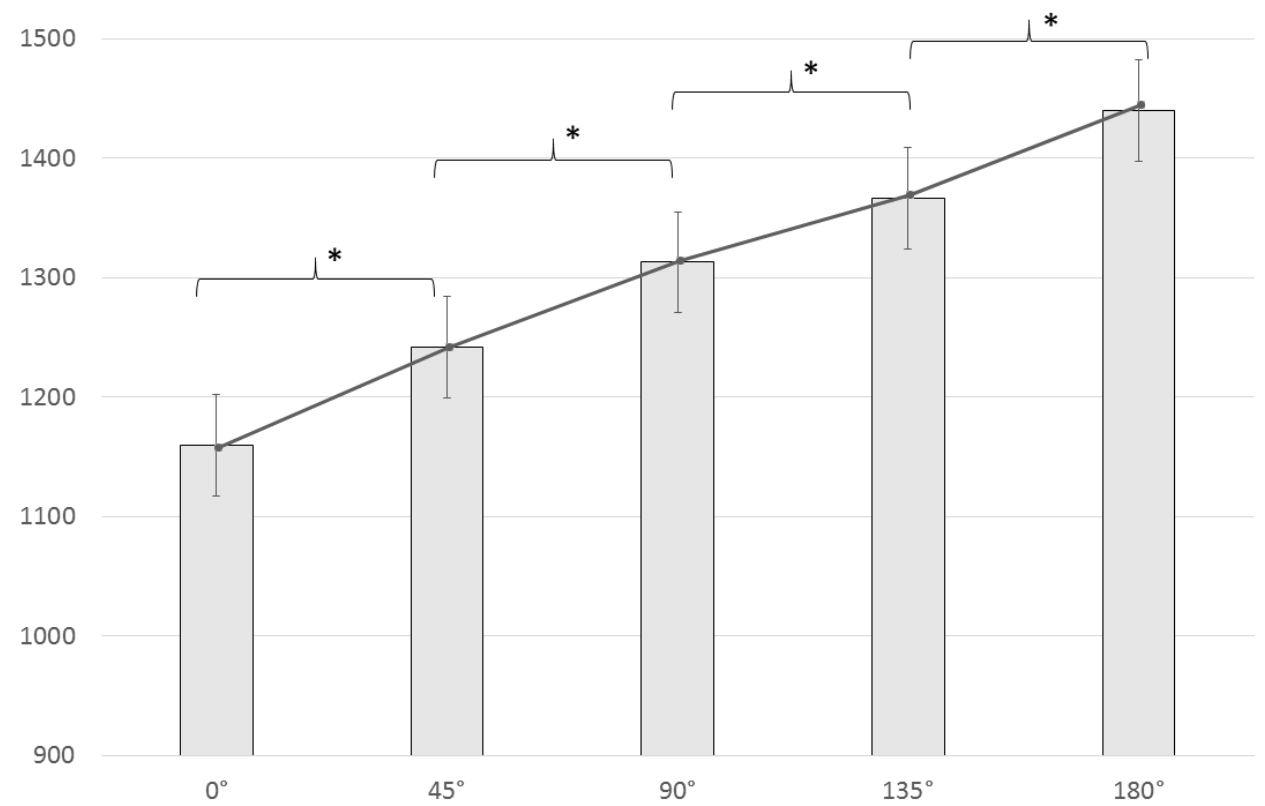

Fig. 6: Mean response times (ms) according to the degree of rotation $\left(0^{\circ}, 45^{\circ}, 90^{\circ}, 135^{\circ}\right.$, and $180^{\circ}$ ). The error bars indicate the $95 \%$ confidence intervals. An asterisk indicates a significant difference $(p<.05)$.

\section{Discussion}

In Experiment 3, we proposed that the orientation of an action is not a crucial parameter in the action-language relationship. Indeed, we suggested that a modification to the orientation could be offset by the use of mental rotation. The aim of this study was to demonstrate that mental rotation is effectively involved when participants perform a task that requires them to recognize rotated PLD. Our results supported this hypothesis. Indeed, a linear relation was observed between the response times of our participants and the degrees of rotation of the PLD, i.e., the more the PLD were rotated, the longer the time required to perform the mental rotations. Thus, the response times were also longer.

\section{General Discussion}


In a previous study, we demonstrated that perceiving a biological human movement facilitates the subsequent processing of a corresponding action verb: a facilitation effect was found when the action performed by a point-light display and the action conveyed by a verb were congruent (Beauprez \& Bidet-Ildei, 2017). We suggested that the activation of sensorimotor representations common to action observation and action language processing could explain this result. The aims of the present study were to replicate this finding and to understand what parameters of an observed action could be responsible of the activation of these sensorimotor representations. In Experiments 1 and 2 (kinematics) and in Experiment 3 (orientation), the facilitation effect was replicated, in that the response times for action verbs were faster when the participants observed prime congruent actions represented by a biological point-light display. This facilitation effect was also found in experiment 3 when the action was performed by a point-light display whose orientation had been modified. However, in Experiments 1 and 2, we demonstrated that important modifications to the action kinematics disturbs the usual link between action observation and action verb understanding, as the facilitation effect disappeared.

As the movement was modified, it might be hypothesized that the existence of the facilitation effect was dependent on the recognition of the movement of the point-light display. However, even if modified PLDs were recognized slightly less often than biological PLDs, the high recognition rates obtained for both the kinematics-modified and the orientation-modified displays do not support this view and do not explain why the facilitation effect was still present when we modified the orientation but disappeared when we modified the kinematics.

Regarding these results, they are more likely to be related to the fact that kinematics is a crucial parameter to activate sensorimotor representations when an action is observed. This agrees with the idea that sensorimotor representations are involved when an observed action 
matches the perceiver's motor repertoire (Calvo-Merino, Glaser, Grèzes, Passingham, \& Haggard, 2005; Martel et al., 2011). Beardsworth and Buckner (1981), for example, observed that participants recognized point-light displays of themselves more easily than they recognized point-light displays of others. In the same vein, Loula and colleagues (2005) demonstrated that one's own motor action are more easily recognized than the action of others. Casile and Giese (2006) showed that training people to walk differently (with a modification in the phases between contralateral articulations) improved their capacity to detect these movements. All these data align with the idea that sensorimotor activation during action perception is linked to the strength of the sensorimotor experience. Interestingly, this effect of motor repertoire can also play an important role in the processing of action words (Lyons et al., 2010). Indeed, Lyons and colleagues (2010) found greater activation in the left premotor cortex when experienced ice hockey players analyzed sentences that involved specific hockey actions than when people without ice hockey experience analyzed the same sentences. Thus, the closer a movement is to our motor repertoire, the stronger the link between action and language is. Conversely, we can hypothesize that the more a movement differs from our motor repertoire, the more difficult it should be to activate sensorimotor representations when we process action verbs. This hypothesis is in accordance with the results obtained in experiment 1 and could explain why the link between action and language was stronger with biological point-light display than with a non-biological point-light display. It also explains why a small change in kinematics (constant condition) did not affect this link whereas a large change (inverted condition) did, since the more the kinematics was modified, the more difficult it must have been to activate sensorimotor representations. Interestingly, the results of Experiment 2 indicated that the influence of action observation on language processing is somatotopic. Indeed, when the kinematics of the part of the body mainly involved in the action were preserved, the link between action observation and action-verb 
processing was preserved, while the link disappeared when the kinematics of this part of the body were modified. Thus, we demonstrated for the first time that the kinematics of an action, and more particularly, the kinematics of the main effector of an action, form a crucial parameter in the activation of sensorimotor representations when an action is observed. However, even though these results should be confirmed in futures studies, they suggest that the activation of action representations is linked to the body part specifically involved in the production of the action.

Concerning the results obtained in Experiment 3, however, it is unlikely that our participants had motor experiences of walking or jumping upside down. However, as explained earlier, we proposed that the facilitation effect observed with orientation-modified point-light displays could be related to the use of mental rotation. The results of Experiment 4 supported this assumption by demonstrating that mental rotation was effectively involved when a task required the recognition of rotated PLD. Thus, a modification to the orientation would not disturb the action-language relationship since it would be compensated by mental rotation. In this case, even if it would require more time, the activation of sensorimotor representations would still be possible, especially because the pattern of kinematics remained the same regardless of display orientation. Consequently, the results obtained in experiment 3 confirm that maintaining biological kinematics is what is truly important to produce the action-language relationship. Globally, these results confirms the idea that the familiarity of the kinematics is a more important factor than the familiarity of the orientation in action processing (Shipley, 2003).

Interestingly, the effect was obtained even if participants mostly reported not having noticed the difference between kinematics-modified displays and biological displays. Therefore, it seems that the modulation of the influence of action observation on language processing is related to implicit mechanisms and does not wholly rely on explicit recognition 
of the action. Perhaps this explicit recognition is necessary, but regardless, it is not sufficient to enable the action-language relationship. This view is in accordance with several experiments showing that kinematic analysis is implicit (Bidet-Ildei et al., 2011; Kandel, Orliaguet, \& Viviani, 2000; Méary, Chary, Palluel-Germain, \& Orliaguet, 2005) and is also in line with brain activation experiments (Willems, Toni, Hagoort, \& Casasanto, 2009). Indeed, in their study, Willems and collaborators asked their participants to perform a lexical decision task with action verbs (implicit process) or to actively imagine performing the actions of the verbs (explicit process). The response patterns obtained in premotor cortex showed that implicit motor simulation during language processing and explicit motor imagery do not engage the same neural substrates. This suggests that the link between action and language is supported by implicit activation of common motor representations (Bidet-Ildei et al., 2017; Bidet-Ildei \& Toussaint, 2015; Zwaan, Taylor, \& de Boer, 2010).

In summary, the results of our study confirm that observation of point-light human actions can modulate action verb processing, and that this effect is likely related to the activation of common sensorimotor representations (Beauprez \& Bidet-Ildei, 2017). Interestingly, we show for the first time that biological kinematics, a key signature of human movements (Johansson, 1973), has a crucial role in the relationship between action and the processing of action verbs. It remains to be determined what mechanisms are at the origin of the decrease in the action-language relationship when the kinematics is non-biological. It could be hypothesized that this decrease relies on inhibition of sensorimotor representations or on modulation of their activation. We consider the second hypothesis more likely: the diminished action-language relationship is related to a weaker degree of activation of sensorimotor representations when the kinematics is non-biological. Indeed, previous studies have shown that language does not mandatorily trigger activity in the motor regions, and some experiments have shown that sensorimotor activation during language are flexible 
(Aravena et al., 2012; Bidet-Ildei et al., 2016). Similar results were found in an fMRI study (van Dam, van Dijk, Bekkering, \& Rueschemeyer, 2012) showing that motor structure recruitment during language hinges on specific conditions, such as the context of the sentence. Thus, these studies provided evidence that language does not trigger mandatorily activity in the motor regions, and that specific parameters of the sentence are able to modulate the activation of sensorimotor representations. In the same manner, we demonstrate here that specific properties of an action, particularly its kinematics, can also modulate the link between action and language, probably by decreasing the activation of sensorimotor representations during action observation. Brain activation studies should be conducted to confirm this explanation. Further research on other parameters of movement would also be necessary to continue to better understand the action-language relationship.

\section{ACNOWLEDGEMENTS}

Support for this research was provided by a grant from La Région Nouvelle Aquitaine (CPER-FEDER P-2017-BAFE-68), in partnership with the European Union (FEDER/ERDF, European Regional Development Fund). This work was a part of the Ph.D. program of Sophie-Anne Beauprez. All the videos used in this paper are available on the PLAViMoP website at http://plavimop.prd.fr. 
Appendix 1: list of verbs

\begin{tabular}{|c|c|c|c|}
\hline \multicolumn{2}{|c|}{ Action verbs } & \multicolumn{2}{c|}{ Non-action verbs } \\
\hline French Verb & English Translation & French Verb & English Translation \\
\hline Applaudir & To applaud & Choisir & To choose \\
\hline Balayer & To sweep & Connaitre & To know \\
\hline Courir & To run & Désirer & To desire \\
\hline Descendre & To get off & Douter & To doubt \\
\hline Gratter & To scratch & Espérer & To hope \\
\hline Marcher & To walk & Etre & To be \\
\hline Monter & To climb & Paraitre & To seem \\
\hline Pédaler & To pedal & Penser & To think \\
\hline Poser & To put down & Pouvoir & To be able \\
\hline Pousser & To push & Raisonner & To reason \\
\hline Ramasser & To pick up & Rêver & To dream \\
\hline Reculer & To move back & Savoir & To know \\
\hline Sauter & To jump & Sembler & To seem \\
\hline Tomber & To fall & Valoir & To be worth \\
\hline Tourner & To turn & Vouloir & To want \\
\hline
\end{tabular}




\section{References}

Aravena, P., Courson, M., Frak, V., Cheylus, A., Paulignan, Y., Deprez, V., \& Nazir, T. A. (2014). Action relevance in linguistic context drives word-induced motor activity. Frontiers in Human Neuroscience, 8. https://doi.org/10.3389/fnhum.2014.00163

Aravena, P., Delevoye-Turrell, Y., Deprez, V., Cheylus, A., Paulignan, Y., Frak, V., \& Nazir, T. (2012). Grip force reveals the context sensitivity of language-induced motor activity during «action words» processing: evidence from sentential negation. PloS One, 7(12), e50287. https://doi.org/10.1371/journal.pone.0050287

Atkinson, A. P., Dittrich, W. H., Gemmell, A. J., \& Young, A. W. (2004). Emotion perception from dynamic and static body expressions in point-light and full-light displays. Perception, 33(6), 717-746. https://doi.org/10.1068/p5096

Aziz-Zadeh, L., \& Damasio, A. (2008). Embodied semantics for actions: Findings from functional brain imaging. Journal of Physiology-Paris, 102(1), 35-39. https://doi.org/10.1016/j.jphysparis.2008.03.012

Aziz-Zadeh, L., Wilson, S. M., Rizzolatti, G., \& Iacoboni, M. (2006). Congruent embodied representations for visually presented actions and linguistic phrases describing actions. Current Biology: CB, 16(18), 1818-1823. https://doi.org/10.1016/j.cub.2006.07.060

Badets, A., Bidet-Ildei, C., \& Pesenti, M. (2015). Influence of biological kinematics on abstract concept processing. Quarterly Journal of Experimental Psychology (2006), 68(3), 608-618. https://doi.org/10.1080/17470218.2014.964737

Bardi, L., Regolin, L., \& Simion, F. (2014). The first time ever I saw your feet: inversion effect in newborns' sensitivity to biological motion. Developmental Psychology, 50(4), 986-993. https://doi.org/10.1037/a0034678

Barsalou, L. W. (1999). Perceptual symbol systems. The Behavioral and Brain Sciences, 22(4), 577-609; discussion 610-660. 
Beardsworth, T., \& Buckner, T. (1981). The ability to recognize oneself from a video recording of one's movements without seeing one's body. Bulletin of the Psychonomic Society, 18(1), 19-22. https://doi.org/10.3758/BF03333558

Beauprez, S.-A., \& Bidet-Ildei, C. (2017). Perceiving a Biological Human Movement Facilitates Action Verb Processing. Current Psychology, 1-5. https://doi.org/10.1007/s12144-017-9694-5

Bertenthal, B. I., \& Pinto, J. (1994). Global Processing of Biological Motions. Psychological Science, 5(4), 221-225.

Bertenthal, B. I., Proffitt, D. R., \& Kramer, S. J. (1987). Perception of biomechanical motions by infants: implementation of various processing constraints. Journal of Experimental Psychology. Human Perception and Performance, 13(4), 577-585.

Bidet-Ildei, C., Gimenes, M., Toussaint, L., Almecija, Y., \& Badets, A. (2016). Sentence plausibility influences the link between action words and the perception of biological human movements. Psychological Research. https://doi.org/10.1007/s00426-016-0776-z

Bidet-Ildei, C., Kitromilides-Salerio, E., Orliaguet, J-P, Badets, A. (2012). Perceptual judgements of handwriting and pointing movements: Influence of kinematics rules. In A. M. Columbus (Ed), Advances in Psychology Research Vol 77, pp 307-316. New York: Nova Publisher.

Bidet-Ildei, C., Kitromilides, E., Orliaguet, J.-P., Pavlova, M., \& Gentaz, E. (2014). Preference for point-light human biological motion in newborns: contribution of translational displacement. Developmental Psychology, 50(1), 113-120. https://doi.org/10.1037/a0032956

Bidet-Ildei, C., Méary, D., \& Orliaguet, J.-P. (2006). Visual Perception of Elliptic movements in 7to-11-year-old children : Influence of Motor Rules. Current Psychology Letters. Behaviour, Brain \& Cognition, (19, Vol. 2, 2006). Consulté à l'adresse http://cpl.revues.org/1071

Bidet-Ildei, C., Meugnot, A., Beauprez, S.-A., Gimenes, M., \& Toussaint, L. (2017). Short-Term Upper Limb Immobilization Affects Action-Word Understanding. Journal of Experimental Psychology. Learning, Memory, and Cognition. https://doi.org/10.1037/xlm0000373 
Bidet-Ildei, C., Sparrow, L., \& Coello, Y. (2011). Reading action word affects the visual perception of biological motion. Acta Psychologica, 137(3), 330-334. https://doi.org/10.1016/j.actpsy.2011.04.001

Bidet-Ildei, C., \& Toussaint, L. (2015). Are judgments for action verbs and point-light human actions equivalent? Cognitive Processing, 16(1), 57-67. https://doi.org/10.1007/s10339-0140634-0

Blake, R. (1993). Cats Perceive Biological Motion. Psychological Science, 4(1), 54-57. https://doi.org/10.1111/j.1467-9280.1993.tb00557.x

Blake, R., \& Shiffrar, M. (2007). Perception of human motion. Annual Review of Psychology, 58, 47-73. https://doi.org/10.1146/annurev.psych.57.102904.190152

Boulenger, V., Roy, A. C., Paulignan, Y., Deprez, V., Jeannerod, M., \& Nazir, T. A. (2006). Cross-talk between language processes and overt motor behavior in the first $200 \mathrm{msec}$ of processing. Journal of Cognitive Neuroscience, 18(10), 1607-1615. https://doi.org/10.1162/jocn.2006.18.10.1607

Bouquet, C. A., Gaurier, V., Shipley, T., Toussaint, L., \& Blandin, Y. (2007). Influence of the perception of biological or non-biological motion on movement execution. Journal of Sports Sciences, 25(5), 519-530. https://doi.org/10.1080/02640410600946803

Calvo-Merino, B., Glaser, D. E., Grèzes, J., Passingham, R. E., \& Haggard, P. (2005). Action observation and acquired motor skills: an FMRI study with expert dancers. Cerebral Cortex (New York, N.Y.: 1991), 15(8), 1243-1249. https://doi.org/10.1093/cercor/bhi007

Casile, A., \& Giese, M. A. (2006). Nonvisual motor training influences biological motion perception. Current Biology: CB, 16(1), 69-74. https://doi.org/10.1016/j.cub.2005.10.071

Chouchourelou, A., Matsuka, T., Harber, K., \& Shiffrar, M. (2006). The visual analysis of $\begin{array}{llll}\text { emotional } & \text { actions. } & \text { Social } & \text { Neuroscience, }\end{array}$ https://doi.org/10.1080/17470910600630599 
Clarke, T. J., Bradshaw, M. F., Field, D. T., Hampson, S. E., \& Rose, D. (2005). The perception of emotion from body movement in point-light displays of interpersonal dialogue. Perception, 34(10), 1171-1180. https://doi.org/10.1068/p5203

Corballis, M. C., \& McMaster, H. (1996). The roles of stimulus-response compatibility and mental rotation in mirror-image and left-right decisions. Can J Exp Psychol, 50(4), 397-401.

Cutting, J. E., \& Kozlowski, L. T. (1977). Recognizing friends by their walk: Gait perception without familiarity cues. Bulletin of the Psychonomic Society, 9(5), 353-356. https://doi.org/10.3758/BF03337021

Davila, A., Schouten, B., \& Verfaillie, K. (2014). Perceiving the direction of articulatory motion in point-light actions. PloS One, 9(12), e115117. https://doi.org/10.1371/journal.pone.0115117

Dehaene, S. (1992). Varieties of numerical abilities. Cognition, 44(1), 1-42. https://doi.org/10.1016/0010-0277(92)90049-N

Fischer, M. H., \& Zwaan, R. A. (2008). Embodied language: a review of the role of the motor system in language comprehension. Quarterly Journal of Experimental Psychology (2006), 61(6), 825-850. https://doi.org/10.1080/17470210701623605

Faul, F., Erdfelder, E., Lang, A.-G., \& Buchner, A. (2007). G*Power 3: a flexible statistical power analysis program for the social, behavioral, and biomedical sciences. Behavior Research Methods, 39(2), 175-191.

Frak, V., Nazir, T., Goyette, M., Cohen, H., \& Jeannerod, M. (2010). Grip Force Is Part of the Semantic Representation of Manual Action Verbs. PLOS ONE, 5(3), e9728. https://doi.org/10.1371/journal.pone.0009728

Hauk, O., Johnsrude, I., \& Pulvermüller, F. (2004). Somatotopic representation of action words in human motor and premotor cortex. Neuron, 41(2), 301-307. 
Hirai, M., Senju, A., Fukushima, H., \& Hiraki, K. (2005). Active processing of biological motion perception: an ERP study. Brain Research. Cognitive Brain Research, 23(2-3), 387-396. https://doi.org/10.1016/j.cogbrainres.2004.11.005

Jirak, D., Menz, M. M., Buccino, G., Borghi, A. M., \& Binkofski, F. (2010). Grasping language--a short story on embodiment. Consciousness and Cognition, 19(3), 711-720. https://doi.org/10.1016/j.concog.2010.06.020

Johansson, G. (s. d.). Visual perception of biological motion and a model for its analysis. Perception \& Psychophysics, 14(2), 201-211. https://doi.org/10.3758/BF03212378

Klepp, A., Weissler, H., Niccolai, V., Terhalle, A., Geisler, H., Schnitzler, A., \& Biermann-Ruben, K. (2014). Neuromagnetic hand and foot motor sources recruited during action verb processing. Brain and Language, 128(1), 41-52. https://doi.org/10.1016/j.bandl.2013.12.001

Kosslyn, S. M., DiGirolamo, G., Thompson, W., \& Alpert, N. (1998). Mental rotation of objects versus hands: neural mechanisms revealed by positron emission tomography. Psychophysiology, 32(2), 151-61.

Kozlowski, L. T., \& Cutting, J. E. (1977). Recognizing the sex of a walker from a dynamic pointlight display. Perception \& Psychophysics, 21(6), 575-580. https://doi.org/10.3758/BF03198740

Kuipers, J.-R., van Koningsbruggen, M., \& Thierry, G. (2013). Semantic priming in the motor cortex: evidence from combined repetitive transcranial magnetic stimulation and event-related potential. Neuroreport, 24(12), 646-651. https://doi.org/10.1097/WNR.0b013e3283631467

Liepelt, R., Dolk, T., \& Prinz, W. (2012). Bidirectional semantic interference between action and speech. Psychological Research, 76(4), 446-455. https://doi.org/10.1007/s00426-011-0390-z

Loula, F., Prasad, S., Harber, K., \& Shiffrar, M. (2005). Recognizing people from their movement. Journal of Experimental Psychology. Human Perception and Performance, 31(1), 210-220. https://doi.org/10.1037/0096-1523.31.1.210 
Lyons, I. M., Mattarella-Micke, A., Cieslak, M., Nusbaum, H. C., Small, S. L., \& Beilock, S. L. (2010). The role of personal experience in the neural processing of action-related language. Brain and Language, 112(3), 214-222. https://doi.org/10.1016/j.band1.2009.05.006

Martel, L., Bidet-Ildei, C., \& Coello, Y. (2011). Anticipating the terminal position of an observed action: Effect of kinematic, structural, and identity information. Visual Cognition, 19(6), 785-798. https://doi.org/10.1080/13506285.2011.587847

Méary, D., Chary, C., Palluel-Germain, R., \& Orliaguet, J.-P. (2005). Visual perception of writing and pointing movements. Perception, 34(9), 1061-1067. https://doi.org/10.1068/p3388

Méary, D., Kitromilides, E., Mazens, K., Graff, C., \& Gentaz, E. (2007). Four-day-old human neonates look longer at non-biological motions of a single point-of-light. PloS One, 2(1), e186. https://doi.org/10.1371/journal.pone.0000186

Mollo, G., Pulvermüller, F., \& Hauk, O. (2016). Movement priming of EEG/MEG brain responses for action-words characterizes the link between language and action. Cortex; a Journal Devoted to the Study of the Nervous System and Behavior, 74, 262-276. https://doi.org/10.1016/j.cortex.2015.10.021

Oram, M. W., \& Perrett, D. I. (1994). Responses of Anterior Superior Temporal Polysensory (STPa) Neurons to «Biological Motion » Stimuli. Journal of Cognitive Neuroscience, 6(2), 99-116. https://doi.org/10.1162/jocn.1994.6.2.99

Orliaguet, J. P., Kandel, S., \& Boë, L. J. (1997). Visual perception of motor anticipation in cursive handwriting: influence of spatial and movement information on the prediction of forthcoming letters. Perception, 26(7), 905-912. https://doi.org/10.1068/p260905

Parron, C., Deruelle, C., \& Fagot, J. (2007). Processing of biological motion point-light displays by baboons (Papio papio). Journal of Experimental Psychology. Animal Behavior Processes, 33(4), 381-391. https://doi.org/10.1037/0097-7403.33.4.381 
Pavlova, M. A., Erb, M., Hagberg, G. E., Loureiro, J., Sokolov, A. N., \& Scheffler, K. (2017). «Wrong Way Up »: Temporal and Spatial Dynamics of the Networks for Body Motion Processing at 9.4 T. Cerebral Cortex (New York, N.Y.: 1991), 27(11), 5318-5330. https://doi.org/10.1093/cercor/bhx151

Pavlova, M., Krägeloh-Mann, I., Birbaumer, N., \& Sokolov, A. (2002a). Biological motion shown backwards: the apparent-facing effect. Perception, 31(4), 435-443. https://doi.org/10.1068/p3262

Pavlova, M., Krägeloh-Mann, I., Birbaumer, N., \& Sokolov, A. (2002b). Biological motion shown backwards: the apparent-facing effect. Perception, 31(4), 435-443. https://doi.org/10.1068/p3262

Pavlova, M., \& Sokolov, A. (2000). Orientation specificity in biological motion perception. Perception \& Psychophysics, 62(5), 889-899.

Pozzo, T., Papaxanthis, C., Petit, J. L., Schweighofer, N., \& Stucchi, N. (2006). Kinematic features of movement tunes perception and action coupling. Behavioural Brain Research, 169(1), 75-82. https://doi.org/10.1016/j.bbr.2005.12.005

Roberts, A., Nguyen, P., Orange, J. B., Jog, M., Nisbet, K. A., \& McRae, K. (2017). Differential impairments of upper and lower limb movements influence action verb processing in Parkinson disease. Cortex; a Journal Devoted to the Study of the Nervous System and Behavior, 97, 49-59. https://doi.org/10.1016/j.cortex.2017.09.022

Runeson, S., \& Frykholm, G. (1981). Visual perception of lifted weight. Journal of Experimental Psychology. Human Perception and Performance, 7(4), 733-740.

Schluessel, V., Kortekamp, N., Cortes, J. A. O., Klein, A., \& Bleckmann, H. (2015). Perception and discrimination of movement and biological motion patterns in fish. Animal Cognition, 18(5), 1077-1091. https://doi.org/10.1007/s10071-015-0876-y 
Shiffrar, M., Lichtey, L., \& Heptulla Chatterjee, S. (1997). The perception of biological motion across apertures. Perception \& Psychophysics, 59(1), 51-59.

Shipley, T. F. (2003). The effect of object and event orientation on perception of biological motion. Psychological Science, 14(4), 377-380.

Simion, F., Regolin, L., \& Bulf, H. (2008). A predisposition for biological motion in the newborn baby. Proceedings of the National Academy of Sciences of the United States of America, 105(2), 809-813. https://doi.org/10.1073/pnas.0707021105

Springer, A., \& Prinz, W. (2010). Action semantics modulate action prediction. Quarterly Journal of Experimental Psychology (2006), $\quad$ 63(11), $\quad$ 2141-2158. https://doi.org/10.1080/17470211003721659

Sumi, S. (1984). Upside-down presentation of the Johansson moving light-spot pattern. Perception, 13(3), 283-286. https://doi.org/10.1068/p130283

Taylor, L. J., \& Zwaan, R. A. (2008). Motor resonance and linguistic focus. Quarterly Journal of $\begin{array}{llll}\text { Experimental Psychology } & \text { (2006), } & \text { 61(6), }\end{array}$ https://doi.org/10.1080/17470210701625519

Tettamanti, M., Buccino, G., Saccuman, M. C., Gallese, V., Danna, M., Scifo, P., ... Perani, D. (2005). Listening to action-related sentences activates fronto-parietal motor circuits. Journal of Cognitive Neuroscience, 17(2), 273-281. https://doi.org/10.1162/0898929053124965

Tomasino, B., \& Gremese, M. (2015). Effects of Stimulus Type and Strategy on Mental Rotation Network: An Activation Likelihood Estimation Meta-Analysis. Frontiers in Human Neuroscience, 9, 693. https://doi.org/10.3389/fnhum.2015.00693

van Dam, W. O., van Dijk, M., Bekkering, H., \& Rueschemeyer, S.-A. (2012). Flexibility in embodied lexical-semantic representations. Human Brain Mapping, 33(10), 2322-2333. https://doi.org/10.1002/hbm.21365 
Viviani, P., \& Stucchi, N. (1989). The effect of movement velocity on form perception: geometric illusions in dynamic displays. Perception \& Psychophysics, 46(3), 266-274.

Willems, R. M., Toni, I., Hagoort, P., \& Casasanto, D. (2009). Neural Dissociations between Action Verb Understanding and Motor Imagery. Journal of Cognitive Neuroscience, 22(10), 2387-2400. https://doi.org/10.1162/jocn.2009.21386

Zwaan, R. A., \& Taylor, L. J. (2006). Seeing, acting, understanding: motor resonance in language comprehension. Journal of Experimental Psychology. General, 135(1), 1-11. https://doi.org/10.1037/0096-3445.135.1.1

Zwaan, R. A., Taylor, L. J., \& de Boer, M. (2010). Motor resonance as a function of narrative time: further tests of the linguistic focus hypothesis. Brain and Language, 112(3), 143-149. https://doi.org/10.1016/j.bandl.2008.11.004 